\title{
Analysis of the Phonon Spectrum Emitted by Superconducting Al-Tunneling Junctions at High Quasiparticle Injection Rates
}

\author{
M. Welte and W. Eisenmenger \\ Universität Stuttgart, Physikalisches Institut, Stuttgart, Federal Republic of Germany
}

Received December 3, 1979; revised version July 31, 1980

\begin{abstract}
The phonon spectrum emitted by superconducting Al-tunneling junctions is analyzed by experiments, in which two junctions of different energy gaps are used as phonon generator and detector. The energy gap of the Al-films is varied by the evaporation conditions. The experiments show clearly that the phonon spectrum and the quasiparticle distributions in the generator are strongly nonthermal and depend markedly on the primary tunneling injection. At high injection rates also significant consequences of gap reductions and instabilities are observed.
\end{abstract}

\section{Introduction}

In the last few years, attention has been given to the phonon spectrum emitted by superconducting tunneling junctions for several reasons: (i) The fact, that the phonon spectrum is well defined and shows sharp structures, makes these junctions to a useful tool in the physics of high frequency phonons [1-6]. (ii) The phonon emission of a superconductor under a finite injection gives a direct clue to the electron-phonon interaction in superconductors [6-10]. (iii) It is possible to drive superconductors far from thermal equilibrium by tunneling injection. The phonon spectrum yields information on the nonequilibrium superconducting state.

Previous work on this subject $[7-10,4,6]$ has clarified the principal properties of the spectrum and the nature of the relevant processes: injection of a definite quasiparticle distribution by tunneling [11], relaxation and recombination of quasiparticles with phonon emission $[12,13]$ and phonon escape and reabsorption [14-19]. Consequently, the emitted phonon spectrum can be described as the sum of the recombination and the relaxation parts. An alternatively useful classification distinguishes between the trapping-influenced contributions and between the primary spectrum resultant from the direct escape of phonons produced in the first decay step of the injected quasiparticles. The experimental investigations yielded the result, that in the case of high phonon trapping and low temperature, i.e., in the case $\tau_{\gamma} / \tau_{p} \gg 1,\left(\tau_{\gamma}^{-1}\right.$ and $\tau_{p}^{-1}$ are the phonon decay constants against escape and reabsorption) the low energy part of the spectrum (phonon energy $\Omega<2 \Delta_{G}, 2 \Delta_{G}$ : energy gap of the generator junction) is significantly determined by the first step relaxation processes $[7,2]$, whereas the high energy part $\left(\Omega \geqq 2 \triangle_{G}\right)$ must be assigned as trapping influenced recombination spectrum [17]. This limit of high recombination phonon trapping applies for superconducting tunneling junctions composed of $\mathrm{Pb}$ - or $\mathrm{Sn}$ films.

The main purpose of this report is to give an experimental analysis of the phonon spectrum generated by a superconducting tunneling diode at high injection rates in the case of low phonon trapping. Under these conditions strong deviations from the predictions of the quasithermal models are to be expected $[10,20,21,23]$. Therefore, the experiments concern the phonon spectra of low impedance Altunneling diodes. Since Al-diodes are also used as detectors with defined frequency dependent response, it is in some cases difficult to distinguish between detector and generator dependent effects. It was, therefore, a further intention of this work to investigate earlier reported detector anomalies $[8,10]$ by new experimental evidence.

\section{Experimental Details}

The experiments have been carried out with an arrangement very similar to that described in earlier 
work [1-5]: two superconducting $\mathrm{Al}-\mathrm{I}-\mathrm{Al}$ tunneling junctions are evaporated on two opposite faces of a dielectric monocrystal; in the most cases a $c$-cut $\mathrm{Al}_{2} \mathrm{O}_{3}$ crystal of $3 \mathrm{~mm}$ thickness and $10 \mathrm{~mm}$ diameter was used. Such a high ratio of thickness to diameter reduces the relative intensity of the sidewall-reflected phonon signal. The crystal and the diodes were directly immersed in the cooling bath of a ${ }^{3} \mathrm{He}$ pumping cryostat, allowing the performance of temperatures down to $0.3 \mathrm{~K}$. In order to suppress DC-Josephson effects, a magnetic field of typically 2 Oe parallel to the diode films was applied.

The Al-films of the diodes were deposited with evaporation rates of $100-200 \AA / s$; in the case of clean vacuum conditions $\left(p_{\text {tot }}<10^{-5}\right.$ Torr) the superconducting parameters of the films turned out to be $2 \Delta_{0}$ $=370 \mu \mathrm{eV}$ and $T_{c}=1.19 \mathrm{~K}$. As usual, the tunneling barrier was prepared by an exposure to pure oxygen having a pressure of 50-300 Torr for a time of 50$300 \mathrm{~s}$. If granular Al-films with enhanced energy gap were desired, oxygen up to $2 \times 10^{-4}$ Torr was added during the evaporation or, alternatively, alumina coated boats were used as evaporation source $[23,24]$. The parameters of the diodes used are listed in Table 1.

The experimental procedure included the determination of the signal dependence on the generator current $i_{D}\left(i_{G}\right)$ and the direct measurement of the differentiated signal $\delta i_{D} / \delta i_{G}$, using convenient modulation techniques. $\left(i_{D}\right.$ and $i_{G}$ are the detector and generator currents. The modulation frequency used was appr. $5 \mathrm{kHz}$. The detector bias voltage was stab-

Table 1. Parameters of the diodes

\begin{tabular}{|c|c|c|c|c|c|}
\hline No. & $\begin{array}{l}\text { Thick- } \\
\text { ness } d \\
(\AA)\end{array}$ & $\begin{array}{l}\text { Area } A \\
\left(\mathrm{~mm}^{2}\right)\end{array}$ & $\begin{array}{l}\text { Energy } \\
\text { gap } \\
2 \Delta \\
(\mu \mathrm{eV})\end{array}$ & $\begin{array}{l}\text { Maximum } \\
\text { current } \\
\text { at } e U=2 A \\
(\mathrm{~mA})\end{array}$ & Substrate \\
\hline $1 \mathrm{a}$ & 5,900 & 2.28 & 370 & 40 & $c-\mathrm{Al}_{2} \mathrm{O}_{3}$ \\
\hline $1 b$ & 5,600 & 2.30 & 370 & 46 & $c-\mathrm{Al}_{2} \mathrm{O}_{3}$ \\
\hline $2 a$ & 5,900 & 2.28 & 370 & 33 & $c-\mathrm{Al}_{2} \mathrm{O}_{3}$ \\
\hline $2 b$ & 5,600 & 2.30 & 370 & 37 & $c-\mathrm{Al}_{2} \mathrm{O}_{3}$ \\
\hline $3 b$ & 4,990 & 1.06 & 370 & 300 & $c-\mathrm{Al}_{2} \mathrm{O}_{3}$ \\
\hline $4 a$ & 2,950 & 1.1 & 370 & 0.75 & $c-\mathrm{Al}_{2} \mathrm{O}_{3}$ \\
\hline $4 b$ & 3,424 & 1.7 & 455 & 8.7 & $c-\mathrm{Al}_{2} \mathrm{O}_{3}$ \\
\hline $8 \mathrm{a}$ & 4,936 & 0.86 & 370 & 5 & $\mathrm{c}-\mathrm{Al}_{2} \mathrm{O}_{3}$ \\
\hline $8 \mathrm{~b}$ & 5,384 & 1.01 & 525 & 23.5 & $c-\mathrm{Al}_{2} \mathrm{O}_{3}$ \\
\hline $9 \mathrm{a}$ & 3,927 & 0.91 & 370 & 3.5 & $c-\mathrm{Al}_{2} \mathrm{O}_{3}$ \\
\hline $9 \mathrm{~b}$ & 2,866 & 0.77 & 400 & 15 & $c-\mathrm{Al}_{2} \mathrm{O}_{3}$ \\
\hline $11 \mathrm{~b}$ & 3,739 & 0.8 & 401 & 65 & $c-\mathrm{Al}_{2} \mathrm{O}_{3}$ \\
\hline $14 a$ & 3,001 & 3.4 & 372 & 8.8 & $c-\mathrm{Al}_{2} \mathrm{O}_{3}$ \\
\hline $14 b$ & 4,115 & 3.4 & 405 & 12.5 & $c-\mathrm{Al}_{2} \mathrm{O}_{3}$ \\
\hline $15 \mathrm{a}$ & 4,500 & 0.92 & 365 & 4.2 & $100-\mathrm{Si}$ \\
\hline $15 \mathrm{~b}$ & 1,200 & 0.99 & 650 & 0.006 & $100-\mathrm{Si}$ \\
\hline $16 a$ & 4,020 & 0.8 & 365 & 82 & $100-\mathrm{Si}$ \\
\hline $16 \mathrm{~b}$ & 4,100 & 0.68 & 540 & 10 & $100-\mathrm{Si}$ \\
\hline
\end{tabular}



Fig. 1. Schematical representation of the singularities of the quasiparticle distribution in a superconductor subjected to tunnelling injection. The same scheme applies in the case that the quasiparticles are generated and redistributed by monochromatic phonons or photons of energy $\Omega_{0}>2 A$, if $e U$ is substituted by $\Omega_{0}$

ilzed electronically in order to avoid that the measured signal voltage $\delta U_{D}=\delta i_{D} \cdot R_{d}$ is affected by changes of the dynamical resistance $R_{d}$ of the detector.) Further, the detector time constants $\tau_{\text {eff }}$ have been measured in order to determine the absolute rate of detected phonons [17].

\section{The Structures of the Primary Spectrum}

The sharp structures in the emitted phonon spectrum are caused by the square root singularities in the BCS density of states. A systematic survey can easily be developed by means of the semiconductor model of the superconductor (Fig. 1): If a superconducting tunneling diode is biased at a generator voltage $U_{G}>2 \Delta_{G} / e$ the injected quasiparticle distribution exhibits a square root singularity at the energies $\pm E=A_{G}, \pm E=e U_{G}-\Delta_{G}, \pm E=e U+\Delta_{G}$, the last singularity is a consequence of the thermal tunneling. (We suppose, that elastic branch scattering is fast in comparison with the inelastic phonon scattering, thus the injected excitation distribution is symmetrical with respect to particle and hole-like excitations.) Within this picture the possible phonon assisted decay processes may be symbolized by arrows connecting particles with free states. An analysis of the density of state influence on the emitted phonon spectrum yields the result, that the transitions from the singularities in the particle distribution to a singularity in the distribution of the free states causes alternatively a step or a logarithmic singularity in the emitted phonon spectrum (Fig. 2). This theoretical prediction shall be illustrated by Fig. 3, showing a calculated phonon spectrum at high injection in the low phonon trapping limit [25]. Cal- 

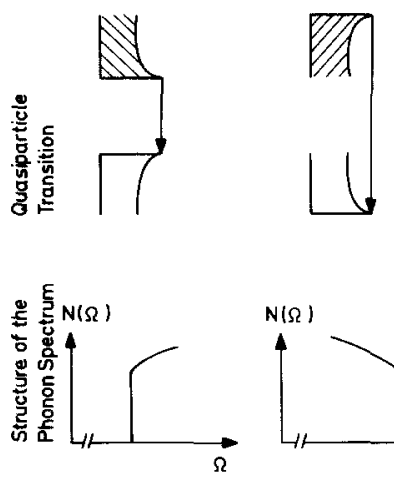

Step

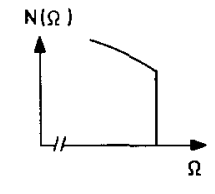

Step

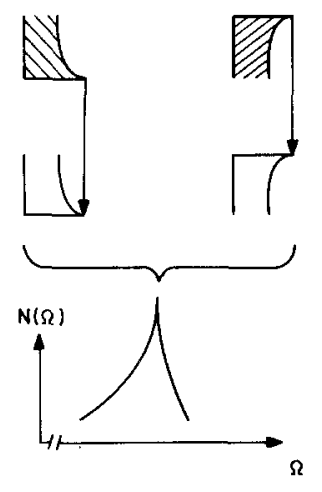

Log. Singularity

Fig. 2. Correspondence between the singularities in the electronic density of states and in the nonequilibrium distribution of the excitations and the resulting structures in the emitted phonon spectrum



Fig. 3. Calculated phonon spectrum at finite injection in the limit $\tau_{\gamma / \tau_{p}}=0$

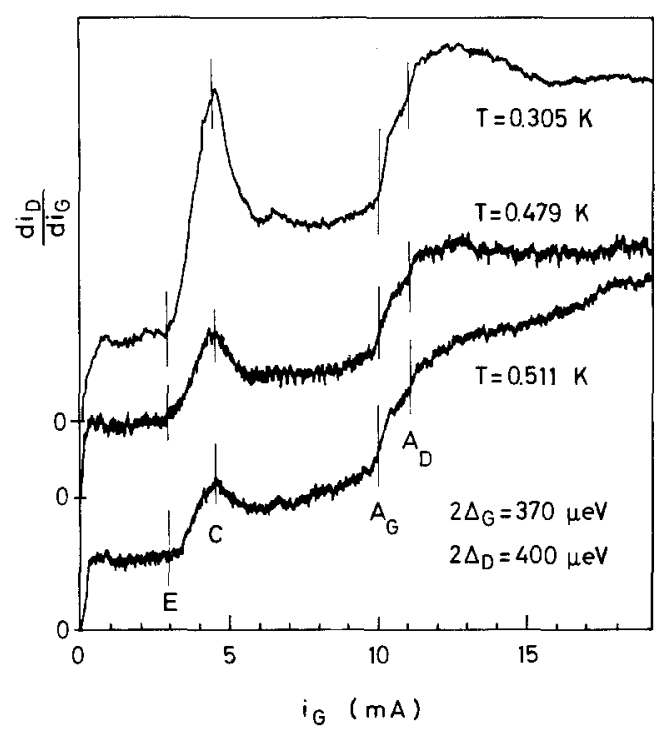

Fig. 4. Differential signal in the case $\Delta_{D} / \Delta_{G}=1.08$ (Diodes $9 \mathrm{a} \rightarrow \mathrm{b}$ )

culated spectra for finite phonon trapping are presented in [26]. According to this analysis the structures, we expect in the primary spectra, are:

$A$ ) a cut-off step in the relaxation spectrum at $\Omega=e U_{G}-2 \Delta_{G}[7]$,

B) a cut-on step in the recombination spectrum at $\Omega=2 \Delta_{G}\left(e U_{G}>2 \Delta_{G}\right)$.

C) At finite quasiparticle density a singularity both in the relaxation and in the recombination spectrum appears at $\Omega=e U_{G}[4]$.

$D)$ A further structure arises only if the injected primary quasiparticle density is not too small compared with the excitation density; i.e; a cut-off step in the recombination spectrum at energy $\Omega=$ $2\left(e U_{G}-\Delta_{G}\right)[26]$.

An essential feature of the structures $A, C, D$ is, that their spectral positions can be tuned by variation of the generator voltage. Due to this, it is possible to demonstrate the existence of these spectral structures by detection of the emitted phonons by means of a detector having an energy gap $2 A_{D}$, which is greater than the generator gap $2 \Delta_{G}[4,5]$. Since such a tunneling diode responds only to phonons with energy $\Omega \geqq 2 \Delta_{D}$, the structures $A, C, D$ must be directly identifiable, if the differentiated signal $\delta i_{D} / \delta i_{G}$ is registrated as a function of the generator current $i_{G}$ or the voltage $U_{G}$. A step- or peak-like structure in the differentiated signal is expected to occur, when the generator voltage fulfills the equation $\Omega_{s}\left(U_{G}\right)=2 \Delta_{D},\left(\Omega_{s}\left(U_{G}\right)\right.$ is the voltage dependent position of the step or of the singularity in the phonon spectrum).

The experimental results are represented in Figs. 4 and 5; they are showing the expected features:

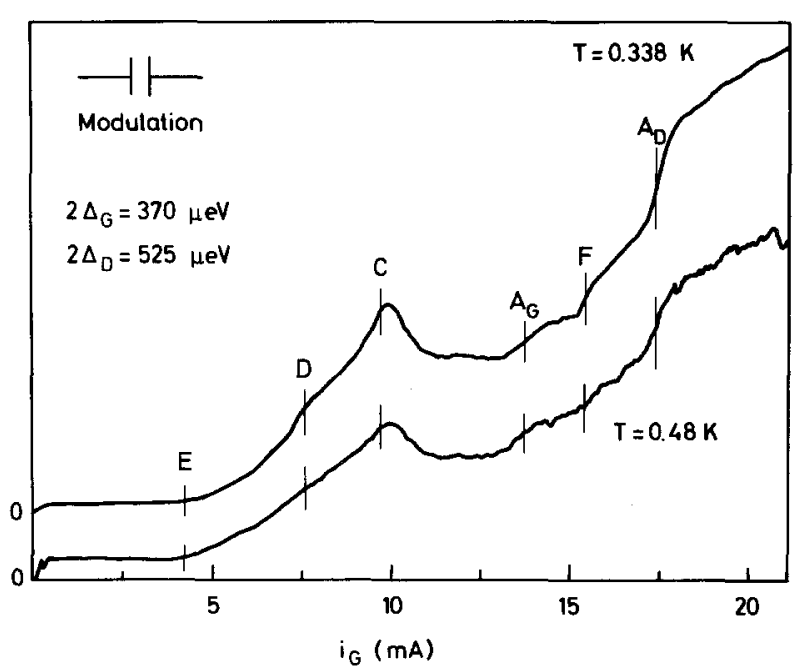

Fig. 5. Signal derivative $d i_{D} / d i_{G}$ in the case $\Delta_{D} / \Delta_{G}=1.42$ (Diodes $8 \mathrm{a} \rightarrow \mathrm{b})$ 


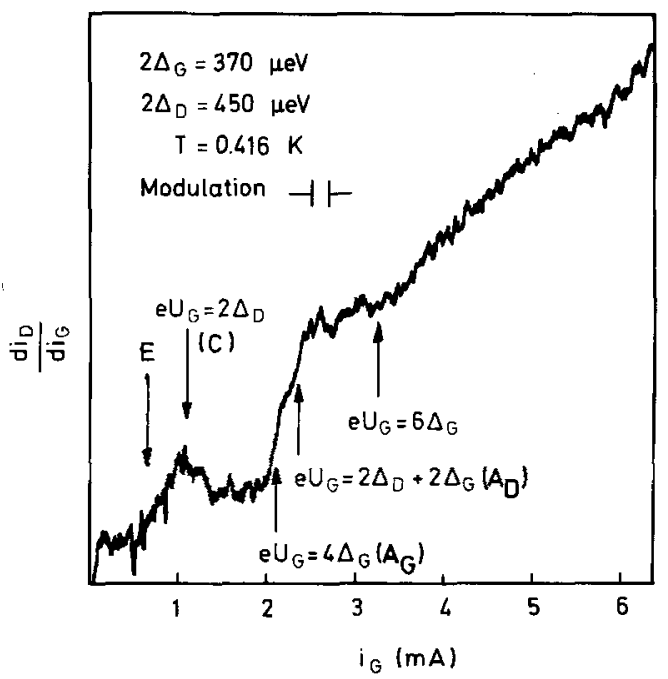

Fig. 6. Signal derivative $d i_{D} / d i_{G}$ for $\Delta_{D}<\Delta_{G}$ in the case of a high impedance generator (Diodes $4 a \rightarrow b$ )

(i) an approximately constant slope of the differential signal in the range where the generator voltage equals $2 \Delta_{G} / e$. (The upper limit of this range is marked by " $E$ "). This signal is due to the high energy tail of the recombination spectrum $\left(\Omega \geqq 2 \Delta_{D}\right)$. The strong temperature dependence indicates that the line width of the recombination spectrum under these injection conditions is determined by thermal phonon scattering. The absolute value of $\delta i_{D} / \delta i_{G}$ in this range is discussed in Sect. $V$.

(ii) Beyond " $E$ " a pronounced peak with maximum at $e U_{G}=2 \Delta_{D}$ follows; in accordance with the analysis given above, this peak is caused by direct recombination of the injected quasiparticles (structure $C$ ). Also the relaxation of excitations injected by thermal tunneling contributes to this structure. (From the fact, that in no case a two peak structure was observed at $e U_{G}=2 A_{D}$, we conclude that the energy gaps of the two Al-films, composing the granular detector junctions, are identical within the finite gap sharpness.) In the case of a great gap difference $\Delta_{D}$ $-\Delta_{G}$ (Fig. 5) this peak shows at the lowest temperature a shoulder at $e U_{G}=\Delta_{G}+A_{D}$, corresponding to the high energy cut-off in the recombination spectrum (Structure $D$ ).

(iii) The high energy cut-off of the relaxation spectrum at $\Omega=e U_{G}-2 \Delta_{G}$ causes two steplike structures " $A_{D}$ " and " $A_{G}$ " in the differentiated signal: at $e U_{G}$ $=2 \Delta_{G}+2 \Delta_{D}$ (Structure $A_{D}$ ) the directly escaping relaxation phonons reach the detection threshold $\Omega \geqq 2 \Delta_{D}$ [4]. But even at the lower voltage $e U_{G}$ $=4 \Delta_{G}$ (Structure $A_{G}$ ) reabsorption in the generator is possible. This reabsorption of relaxation phonons behaves as an additional injection source, increasing the emission rate of recombination phonons, which fulfill partly the detection condition $\Omega \geqq 2 \Delta_{D}$. (It must be noted, that only in the case of high impedance generator junctions the Structures " $A_{D}$ " and " $A_{G}$ " appear exactly at $e U_{G}=2 \Delta_{G}+2 \Delta_{D}$ or $e U_{G}$ $=4 \Delta_{G}$ (Fig. 6); for low impedance generators the injection induced gap reduction must be taken into account.)

(iv) In Fig. 5 the low temperature measurement shows an additional structure, which can be assigned to the generator voltage $e U_{G}=3 \Delta_{G}+\Delta_{D}$ (Point $F$ ). Indeed, there exists a process, which yields detectable phonons $\left(\Omega \geqq 2 \Delta_{D}\right)$ starting at this bias threshold: The maximum energy of the excitations created by reabsorption of relaxation phonons is $E_{\max }=e U_{G}$ $-3 \Delta_{G}$. By recombination a spectral component is produced with maximum phonon energy $\Omega=2\left(e U_{G}\right.$ $\left.-3 \Delta_{G}\right)$. An analytical discussion shows, that the additional signal produced by these phonons has approximately the slope

$\frac{\delta i_{D}}{\delta i_{G}} \mid$ addit. $\sim\left|\frac{e U_{G}-\left(3 \Delta_{G}+\Delta_{D}\right)}{e U_{G}-4 \Delta_{G}}\right|^{2}$

$\left(e U_{G}>3 \Delta_{G}+\Delta_{D}\right)$.

It is obvious, that the square law onset is sharpened by the denominator. Therefore, the proposed interpretation of the relatively sharp structure appears to be consistent with the measurement. As an alternative process in considering the decay of excitations with energy $E>3 \Delta$ also the possibility of phononless decays must be taken into account $[13,27]$. Calculations showed [25] that the secondary quasiparticle distributions produced in such processes are qualitatively similar to those produced by relaxation and subsequent reabsorption. Therefore, the decision between these two decay processes necessitates more detailed calculations and an increased measuring accuracy.

\section{Influence of the Phonon Polarization}

In this section, we discuss the fact, that the relaxation phonon contribution detected in experiments with $\Delta_{G}>\Delta_{D}$ can be subject to changes in the ratio of longitudinal and transverse phonons. Due to the different propagation properties in the anisotropic substrate, such changes should influence the detected intensity. In order to be definite, we first develop a theoretical model describing this effect, then we present the corresponding analysis for Al-diodes.

We suppose an isotropic electron-phonon interaction. Within this model the emission probabilities for longitudinal and transverse phonons have a constant ratio $g_{l} / g_{t}$ for each electronic decay step. The 
ratio of the phonon mean free paths in the superconductor is then given by:

$$
\Lambda_{l} / \Lambda_{t}=\left(c_{t}^{2} \cdot g_{t}\right) /\left(2 c_{l}^{2} \cdot g_{l}\right)
$$

( $c$ is the velocity of sound). In applying this to a superconducting film having a thickness $d \gg \Lambda_{\sigma}$ $(\sigma=l, t)$, the well-known result is yielded [6], that the relative values of the phonon fluxes of different polarizations against the boundaries of the generator are independent from the interaction parameters $g_{l} / g_{t}$ or $\Lambda_{l} / \Lambda_{t}$ :

$F_{G l}: F_{G t}=c_{t}^{2} /\left(2 c_{l}^{2}\right)$

( $F_{G \sigma}$ : flux density of one polarization). In the opposite limiting case $\left(\Lambda_{\sigma} \gg d\right)$ the corresponding result is:

$F_{G l} / F_{G t}=g_{l} \bar{T}_{t G} /\left(g_{t} \bar{T}_{l G}\right), \quad \bar{T}_{\sigma G}=\bar{T}_{\sigma G S}+\bar{T}_{\sigma G H e}$

i.e., the phonon fluxes are determined by the internal interaction strength as well as be the phonon transmissions $\bar{T}_{\sigma G S}$ and $\bar{T}_{\sigma G H e}$ of the two boundaries of the generator to the substrate and to the helium bath, which are averaged over all angles of incidence $[18,19]$.

In time-resolved measurements, the intensity of longitudinal and transverse phonon signals may be determined by time of flight separation of the polarizations [28]. In working with Al-detectors at low temperatures, this method becomes difficult, as a consequence of the relatively long detector time constants $(5-30 \mu \mathrm{s})$. Nevertheless, the detector signal should markedly depend on the phonon polarization, since the phonon rate $\dot{n}_{D}$ entering the detector is related to $F_{G \sigma}$ by [28]

$\dot{n}_{D}=\dot{n}_{D l}+\dot{n}_{D t}$

$\dot{n}_{D \sigma}=T_{\sigma \perp D} A_{D} \cdot f_{\sigma} A_{G} \cdot \bar{T}_{\sigma G S} \cdot F_{G \sigma} /\left(\pi \cdot r_{G D}^{2}\right)$

$\left(f_{\sigma}\right.$ : phonon focussing factor [29], $A_{G D}$ : generator or detector area, $r_{G D}=$ distance generator - detector, $T_{\sigma \perp D}$ : transmission of the boundary substrate - detector for perpendicular incidence).

A critical test of these predictions is possible in using the tunability of the cut-off energy $\Omega=e U_{G}$ $-2 A_{G}$ in the relaxation spectrum. In determining the differentiated signal $\delta i_{D} / \delta i_{G}$, the contribution of the primarily emitted relaxation phonons may be considered as a modulation isolated monochromatic phonon signal of energy $\Omega=e U_{G}-2 \Delta_{G}$ [2]. Thereby, for $e U_{G}<4 \Delta_{G}$ the condition $\Lambda_{\sigma} \gg d$ is fulfilled and (3) applies. On the other hand, in the regime $e U_{G} \geqq 4 \Delta_{G}$ the monochromatic relaxation signal may be reabsorbed and for junctions of thickness $d \gtrsim 1000 \AA$ the ratio of phonon fluxes in governed by (2). Thus, detecting both polarization components simultaneously in experiments with $\Delta_{G}>\Delta_{D}$ a steplike change in the modulated signal at $e U_{G}=4 \Delta_{G}$ must be expected as a consequence of the predicted change of the mode ratio; i.e.; the relaxation phonon signal $\dot{n}_{D}$ should be changed at $e U_{G}=4 \Delta_{G}$ by the factor $M=\dot{n}_{D<} / \dot{n}_{D>}$, which can be calculated form (2) - (4) under the following assumption:

$F_{G l<}: \bar{T}_{l G}+F_{G t<} \cdot \bar{T}_{t G}=F_{G l>} \cdot \bar{T}_{l G}+F_{G t>} \cdot \bar{T}_{t G}$

(The indices $<,>$ refer to $\gtrless 4 \Delta_{G}$ and to the low or high phonon trapping limit). This condition is due to the premse, that all modulation isolated relaxation phonons for frequency $\Omega$ escape into the substrate or into the helium either directly $\left(\Omega<2 \Delta_{G}\right)$ or after being converted into $2 \Delta_{G}$-phonons by reabsorption $\left(\Omega>2 \triangle_{G}\right)$.

The numerical evaluation of the model was performed for a $c-\mathrm{Al}_{2} \mathrm{O}_{3}$ and a 100 -Si substrate, each of a thickness of $3 \mathrm{~mm}$, and the area of the detector and generator junction was $1 \mathrm{~mm}^{2}$. The transmissions of the metal-insulator interfaces were determined according to the acoustical mismatch theory [18], [19] and using a transmission parameter $\bar{T}_{G H e}=0.2$ we supposed that the anomalous transmission of a metal- ${ }^{3} \mathrm{He}$ interface for $90-\mathrm{GHz}$ phonons is not very different from that of a ${ }^{4} \mathrm{He}$ interface for $280-\mathrm{GHz}$ phonons $[17,41]$. The phonon focusing factors for this geometry are $f_{l}=0.44, f_{t}=2.46(100-\mathrm{Si}[30])$ and $f_{l}=1.98, f_{t}=0.69\left(c-\mathrm{Al}_{2} \mathrm{O}_{3}\right.$ [31]). Supposing a ratio $\Lambda_{l} / \Lambda_{t}=0.47$ according to the analysis of Long [16], the resulting values are $M=0.86\left(c-\mathrm{Al}_{2} \mathrm{O}_{3}\right)$ and $M=1.1(100-\mathrm{Si})$.

In analyzing the relaxation phonon signal at $e U_{G}$ $=4 \Delta_{G}$, also the possibility of enhanced volume losses must be discussed. In the high injection limit, the dominant source of such volume losses is the inelastic phonon quasiparticle scattering, since the secondary phonons, reemitted by relaxation of the excited quasiparticles may have a smaller energy than the originally absorbed phonons. But since the time constants $\tau_{\gamma}^{-1}, \tau_{V}^{-1}$ for phonon decay by escape and by inelastic quasiparticle scattering are continuous functions of the phonon energy, the ratio of the corresponding decay rates $R_{\gamma} / R_{V}=\tau_{\gamma}^{-1} / \tau_{V}^{-1}$ remains constant, if the phonon energy is tuned over the pairbreaking threshold $\Omega=2 \Delta$. Due to this, these volume losses cannot cause a steplike structure in the differentiated signal $\delta i_{G} / \delta i_{D}$ at $e U_{G}=4 A_{G}$ $\left(\Delta_{G}>\Delta_{D}\right)$.

In agreement with this discussion, in all experiments with Al-diodes on a sapphire substrate $(c$-cut) we observed a reduction of the differentiated signal at $e U_{G}=4 \Delta_{G}$ in the case $\Delta_{G}>\Delta_{D}$ (Fig. 7). The measured values of signal considered in comparison to the step 


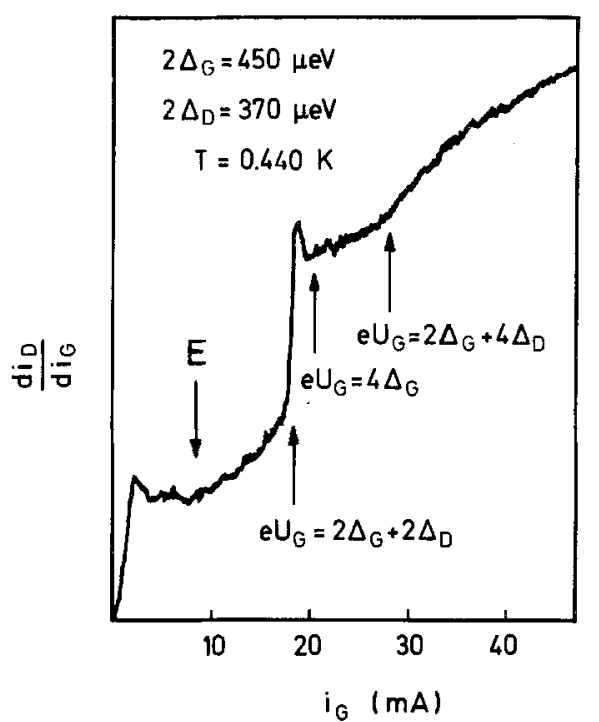

Fig. 7. Signal derivative $d i_{D} / d i_{G}$ for $A_{G}>A_{D}$ (Diodes $4 \mathrm{~b} \rightarrow \mathrm{a}$ ). The substrate is $\mathcal{c}-\mathrm{Al}_{2} \mathrm{O}_{3}$

at $e U_{G}=2 \Delta_{G}+2 \Delta_{D}$ correspond to values of $M_{\text {exp }}$ $=70-80 \%$. In order to confirm the interpretation by a change in the emitted mode ratio, similar measurements with a 100 -orientated Si-substrate have been carried out; in contrast to the $c$-sapphire this substrate favors the transverse polarized phonons above the longitudinal mode. In the experiments with generators, evaporated in an oxigen atmosphere (1-2 $10^{-4}$ Torr), resulting in very high impedance diodes, only one dominant step at $e U_{G}=4 \Delta_{G}$ was found, and within the signal to noise ratio no significant structure at $e U_{G}=2 \Delta_{G}+2 \Delta_{D}$ could be identified (Fig, 8) indicating $M_{\exp }>3,5$. In contrast to this result, only a step at $e U_{G}=2 \Delta_{G}+2 \Delta_{D}$ and no structure at $e U_{G}=4 \Delta_{G}$ was found when the Al-generator was prepared by evaporation from an alumina coated boat. (Fig. 9)

A consistent interpretation of these surprisingly different results requires to take into account elastic mode converting scattering in the generator film. Under the premise of a short time constant $\tau_{c}$ for these scattering processes $\left(\tau_{\gamma} / \tau_{c} \gg 1\right)$, the ratio $F_{G l} / F_{G t}$ equals (2) independently of $\Lambda_{\sigma} / d$. Moreover, the value of $\tau_{c}$ can strongly be determined by the microcrystalline composition of the film; this explains the distinct influence of the evaporation conditions and of the substrate material and the absence of any structure at $e U_{G}=4 \Delta_{G}$ in the case of Fig. 9. The assumption of strong mode conversion by elastic scattering is further confirmed by the fact, that also in similar experiments $\left(A_{G}>A_{D}\right)$ with $\mathrm{Sn}$ and $\mathrm{Pb}$ generators no change in the intensity of longitudinal and transverse phonons could be observed when the relaxation phonon energy is tuned over the reabsorp- tion threshold $[2,32]$. (In time-resolved measurements with Sn-generators signal changes at $e U_{G}$ $=4 A_{G}$ were reported [44], which are consequences of the finite excape time of the phonons in the generator. This interpretation of the $4 \Delta_{G}$-structure cannot be applied to our measurements on Al-junctions, since we used modulation frequencies $f \cong 5 \mathrm{kHz}$, which were small compared to the invese time constant $\tau_{d}^{-1}$ of the generator under $D C$-injection, which we estimate to be $\tau_{d}<1 \mu \mathrm{s}$, comp. Sect. VI).

Considering the observed values of $M_{\text {exp }}$, the measurements with a $c-\mathrm{Al}_{2} \mathrm{O}_{3}$ substrate suggest a ratio of $\Lambda_{V} / \Lambda_{t} \simeq 0.27$. On the other hand, the value of $M_{\exp } \geqq 3.5$, as derived from the measurement on 100 $\mathrm{Si}$, cannot be explained within the presented model, which assumes a polycristalline film structure with isotropic orientation distribution. It must be further noted, that the measurements discussed above are performed with generators having different energy gaps. This indicates the difference in the degree of film disturbance by impurities, etc., which may be also of influence on the ratio of transverse and longitudinal phonon intensities. In addition, it is known, that phonons decay at the metal - sub-

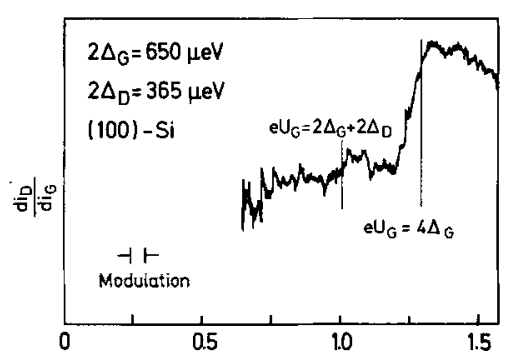

Fig. 8. Signal derivative $d i_{D} / d i_{G}$ for $\Delta_{G}>\Delta_{D}$ in the range $e U_{G}$ $=2 \Delta_{D}+2 \Delta_{G}$ and $e U_{G}=4 \Delta_{G}$. The substrate is (100)-Si. The generator diode was prepared by evaporation from an alumina coated Mo boat (Diodes $15 \mathrm{~b} \rightarrow \mathrm{a}$ )

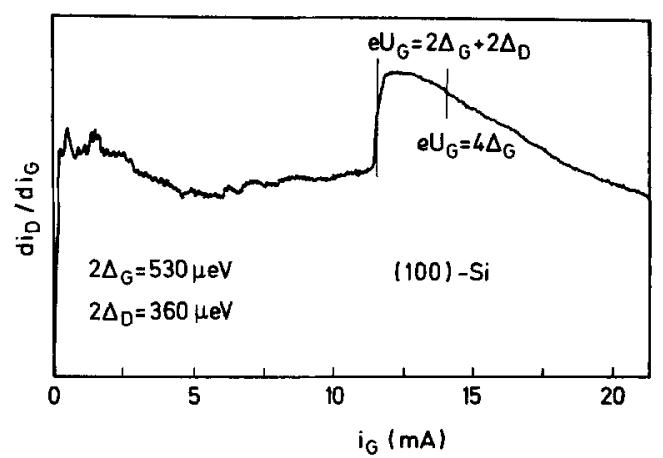

Fig. 9. Signal derivative $d i_{D} / d i_{G}$ for $\Delta_{G}>\Delta_{D}$ and (100)-Si substrate. The generator diode was prepared by evaporation from a tungsten boat and oxygen was added during evaporation (Diodes $16 \mathrm{~b} \rightarrow \mathrm{a})$ 
strate boundary to a significant percentage up to $65 \%$. The decay is different for longitudinal and transverse modes and depends on the method of substrate surface preparation $[28,34]$.

\section{Influence of Gap Reduction and Gap Instability}

The low impedance generator diodes, we used, showed pronounced effects of injection induced gap reduction and gap instabilities. As to the gap reduction, theory predicts for weak coupling superconductors [35]

$$
\begin{aligned}
& -\ln \left(\Delta / \Delta_{0}\right)=2 \int_{\Delta}^{\infty} d E f(E) /\left(E^{2}-\Delta^{2}\right)^{1 / 2} \\
& \Delta_{0}=\Delta(T=0)
\end{aligned}
$$

Under the premise, that the quasiparticle energies can be approximated by $E \simeq \Delta_{G}$ the gap reduction is given by $[20]$ :

$\delta \equiv\left(\Delta_{0}-\Delta\right) / \Delta_{0}=\rho /\left(2 N_{0} \Delta_{0}\right) \quad(\delta \ll 1)$

( $\rho$ : quasiparticle density). Using the rate equations of Rothwarf and Taylor [15] for the overinjection case and assuming that the quasiparticles generated by injection are homogeneously distributed in the diode volume $V$, it follows:

$\delta^{2}=\frac{\pi i_{G}\left(1+\tau_{\gamma} / \tau_{p}\right) \cdot \tau_{p}}{4 e V \Delta^{3} P}$

The parameter $P$ describes the phonon density of states $D(\Omega)=P \cdot \Omega^{2}$. For $4 d / A>1$ the escape time constant $\tau_{\gamma}$ can be approximated by [17]:

$1+\tau_{\gamma} / \tau_{p}=4 d / A \cdot\left(\bar{T}_{G H e}+\bar{T}_{G S}\right)$

and consequently

$\delta^{2}=\pi \cdot i_{G} / e A c P\left(\bar{T}_{G H e}+\bar{T}_{G S}\right)$

We note further, that the above approximation applies only if the surface losses, as described by $\tau_{\gamma}$ are the dominant loss source for the recombination phonons. This premise does not apply in the limit of both, high phonon trapping $\left(\tau_{\gamma} / \tau_{p} \gg 1\right)$ and high quasiparticle density. In this case the inelastic phonon quasiparticle scattering can exceed the surface losses [36].

The gap reductions have been determined in two ways: directly from $i-U$-characteristics of the diodes in the range $e U=2 \Delta$ and, moreover, in the phonon transmission - detection experiments by the observation of structures, which must be assigned to generator voltages like $e U_{G}=2 \Delta_{G}\left(i_{G}\right)+2 \Delta_{D 0}$ or $e U_{G}$
Table 2. The gap reductions determined from the structures in the differentiated signal $\delta i_{D} / \delta i_{G}$

\begin{tabular}{llllll}
\hline Diode & Structure $\begin{array}{l}i \\
(\mathrm{~mA})\end{array}$ & $\begin{array}{l}2 \Delta \\
(\mu \mathrm{eV})\end{array}$ & \multicolumn{2}{c}{$\delta(\%)$} & \multicolumn{2}{c}{\begin{tabular}{l} 
exp \\
\cline { 5 - 6 }
\end{tabular}} & & & & calc \\
\hline $1 \mathrm{a}$ & $A_{G}$ & 120 & 320 & 13.5 & 14.3 \\
$1 \mathrm{~b}$ & $A_{G}$ & 111 & 315 & 14.9 & 13.7 \\
$2 \mathrm{a}$ & $A_{G}$ & 100 & 315 & 14.9 & 13.0 \\
$2 \mathrm{~b}$ & $A_{G}$ & 75 & 300 & 19.0 & 11.1 \\
$3 \mathrm{a}$ & $A_{G}$ & 82 & 330 & 10.8 & 11.8 \\
$8 \mathrm{a}$ & $A_{G}$ & 13.7 & 355 & 4.0 & 7.8 \\
$8 \mathrm{~b}$ & $A_{G}$ & 50.5 & 500 & 4.7 & 6.9 \\
$9 \mathrm{a}$ & $A_{G}$ & 11 & 365 & 1.3 & 6.8 \\
$9 \mathrm{~b}$ & $A_{D}$ & 39 & 370 & 7.5 & 12 \\
\hline
\end{tabular}

$=4 \Delta_{G}\left(i_{G}\right)$. The gap reductions, as determined by the second method, are listed in Table 2; the calculated values $\delta_{\text {calc }}$ given therein are determined according to (10) with $\bar{T}_{G H e}=0.2$ (compare Sect. IV). This last assumption $\left(\bar{T}_{G H e} \simeq 0.2\right)$ is confirmed by the good agreement between experimental and theoretical values (diodes 1a, 1b, 2a, 3a). The deviations in the case of diodes $8 \mathrm{a}, 8 \mathrm{~b}, 9 \mathrm{~b}$ can be explained by the possibility of an enhanced surface transmission from the metal film into the He-bath due to adsorbed impurities [28].

On the other hand, only in the $i-U$ characteristics of diodes with pure Al-films a clear gap reduction could be observed at $e U=2 A$ (Fig. 10). Further, none of the prepared diodes showed the parabolic slope, as predicted by (9). The origin of these discrepancies must be explained by the following possible causes: (i) the premise of a homogeneous quasiparticle density is probably violated, especially at $e U$ $=2 \Delta[38,39]$. (ii) The measured $\Delta$ - and $\Delta_{0}$-values are mean values which are affected by finite gap sharpness and possibly also by gap inhomogeneities. (iii) Injecting just at $2 A$, the distribution of the recombination phonons can be sharply peaked at $\Omega$ $=2 \Delta$. Due to the finite gap sharpness, the phonon mean free path and consequently also the trapping factor $\tau_{y} / \tau_{p}$ depends sensitively on the mean frequency of the recombination phonons (compare Sect. IV).

In addition, discontinuous gap reductions in the range of $e U=2 \Delta$ are a common feature of the characteristics of low impedance Al-diodes; i.e., of Al-diodes with a maximum current density i(eU $=2 \Delta) / A \gtrsim 10 \mathrm{~mA} / \mathrm{mm}^{2}$. This gap switching occurred reproducible and was markedly dependent on the preparation conditions of the films (Fig. 10): Pure junctions $\left(2 \Delta_{0}=370 \mu \mathrm{eV}\right)$ showed the gap switching at $e U=2 A$ in the low injection range (Fig. 10a, b). In the case of Al-films with slightly enhanced gap 

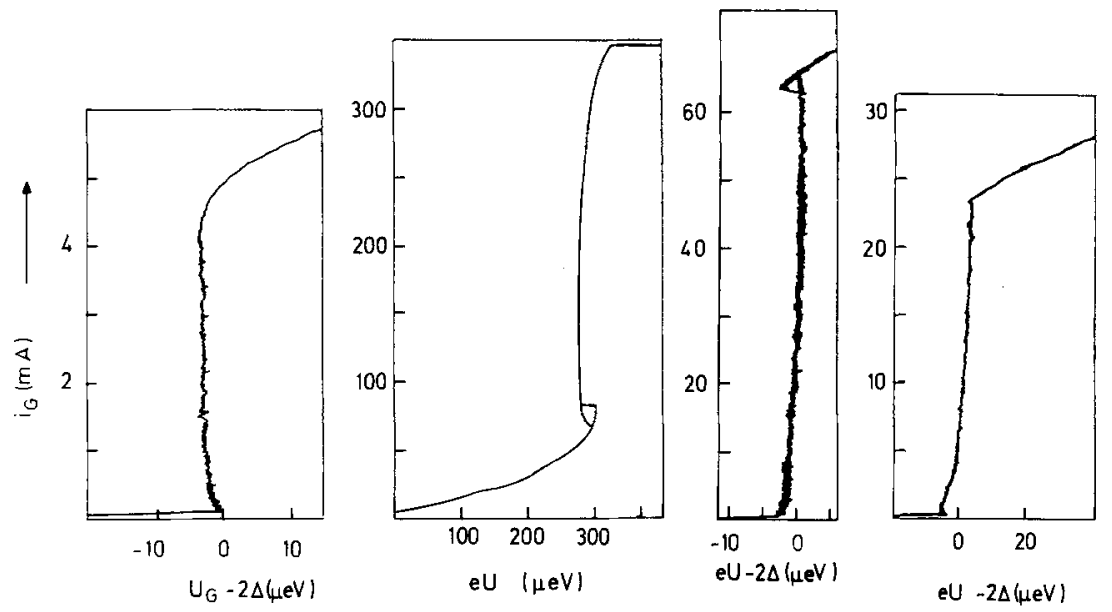

Fig. 10. $i-U$-characteristics in the regime $e U$ $=2 \Delta$. a), b): junctions with pure Al-films, low and extremely low impedance case. c), d): junctions with granular Al-films, slightly and strongly enhanced energy gaps. (Diodes 8a, 3b, 11b, 8b)
$(2 \Delta=400 \mu \mathrm{eV})$ a corresponding discontinuity occurs at the upper end of the vertically rising part of the diode characteristics (Fig. 10c). Junctions with strongly enhanced gap $(2 \Delta \cong 520 \mu \mathrm{eV})$ show at this point a sharp kink in the slope of the characteristics (Fig. 10d); this seems to be a similar gap switching as in Fig. 10c, but without any hysteresis.

From this it appears that the most plausible explanation of these effects is the assumption of an inhomogeous state [37-39], but it is difficult to decide whether this state is caused by an injection induced instability of the superconducting state $[37,38]$ or is due preparation conditioned inhomogeneities of the films. It must further be taken into account, that in biasing the diode near or just below $e U=2 \bar{\Delta}(\bar{\Delta}$ : average gap value), quasiparticles are only injected into the low gap regions and, consequently only there the energy gap is further reduced. Therefore, an inhomogeneity both of the injection induced and of the preparation conditioned type may be enhanced by the injection conditions in a tunneling diode biased at $e U \simeq 2 \Delta$ [39].

As to the strong influence of the preparation conditions of the films on the type of the gap instability, we note, that the electronic mean free paths $l$ and also the coherence lengths $\xi$ can be estimated by $\xi \simeq l \simeq 2000 \AA \quad(2 \Delta=370 \mu \mathrm{eV}$, pure Al-films $)$ and $\xi \simeq l \simeq 400 \AA(2 \Delta=400 \mu \mathrm{eV}$, granular films) $[23,24$, $40]$. It follows from these values that in undisturbed films of thickness $d \simeq 2000 \AA$ the energy gap $\Delta$ can only vary within the film plane; however, a gap dependence on the distance to the tunneling barrier is possible in granular films. There are two indications supporting the assumption of a gap reduction near the tunneling barrier in junctions consisting of granular Al-films: (i) the difference in the coherence lengths explains the two types of gap instabilities. (ii) If $\mathrm{Al}$-tunneling diodes are held at room temperature for some hours, the asymptotic resistance $R_{\infty}$ increases. This indicates that oxygen atoms gettered during evaporation diffuse at room temperature towards the oxide layer, where they form stable $\mathrm{Al}_{2} \mathrm{O}_{3}$. Consequently, the layers of the film near the tunneling barrier can develop a reduced oxigen content and a smaller energy gap. It seems, therefore, to be plausible that the inhomogeneous state of granular Al-diodes under injection is primarily caused by the preparation conditions.

Under the premise of such a layer inhomogeneity, the gap switching of the granular diodes (Fig. 10c) may be explained in a two-gap model: It is assumed, that the diode is composed of two inner layers with an energy gap $2 \Delta^{-}$and a total thickness $d^{-}$and two outer layers with an enhanced gap $2 \Delta^{+}$and a total thickness $d^{+}$. At $e U=2 \Delta^{-}$quasiparticles are only injected into the low gap regions near the tunneling barrier. Provided that the resulting recombination phonon spectrum is sharply peaked at $\Omega=2 \Delta^{-}$, the escape of these phonons into the high gap regions is neither hindered by reabsorption nor by acoustical mismatch; i.e. the resulting phonon escape time $\tau_{\gamma}^{-}$ will appreciably be shorter than for homogeneous diodes $\left(\tau_{\gamma}^{+}\right)$which applies in the bias regime $e U>2 \Delta^{+}$. According to (8), the gap reduction $\delta^{+}$in the second case is greater than $\delta^{-}$if $\tau_{\gamma}^{+} / \tau_{\gamma}^{-}>d^{+} / d^{-}$ and hysteresis is possible if $\Delta_{0}^{+}\left(1-\delta^{+}\right)<\Delta^{-}$ $\left(1-\delta^{-}\right)$.

In contrast to the case of granular Al-films, the gap switching of pure junctions (Fig. 10a, b) is found to be strongly influenced by the applied magnetic field and also by flux trapped in the films [38]. These effects being subject of further experimental investigation are possibly an indication that the gap instability in these diodes is not due to intrinsic inhomogeneities of the films but to magnetic flux and injection. 


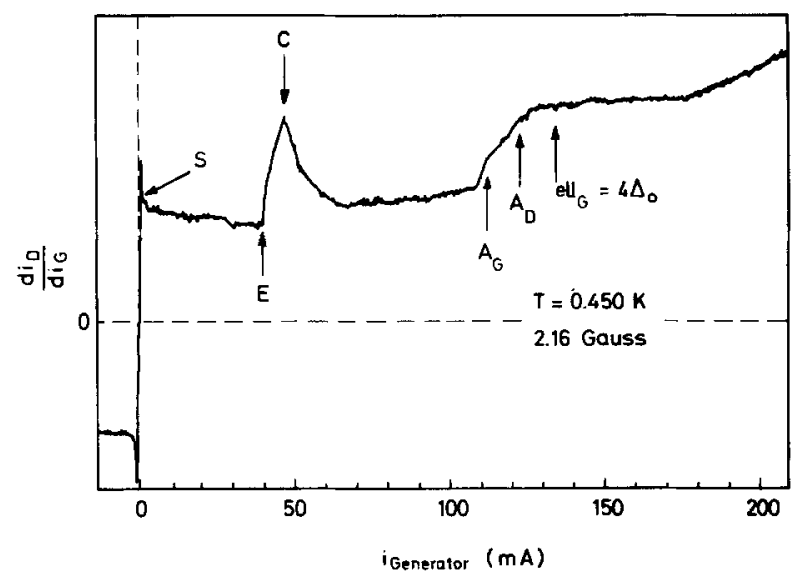

Fig. 11. Signal derivative $d i_{D} / d i_{G}$ for $\Delta_{D_{0}}=\Delta_{G_{0}}$ and low impedance generator. The signal reduction due to the gap switching is assigned by $S$ (Junctions $1 \mathrm{a} \rightarrow \mathrm{b}$ ). If generator and detector junctions are interchanged (i.e., in the case $1 b \rightarrow a$ ), the result is qualitatively identical

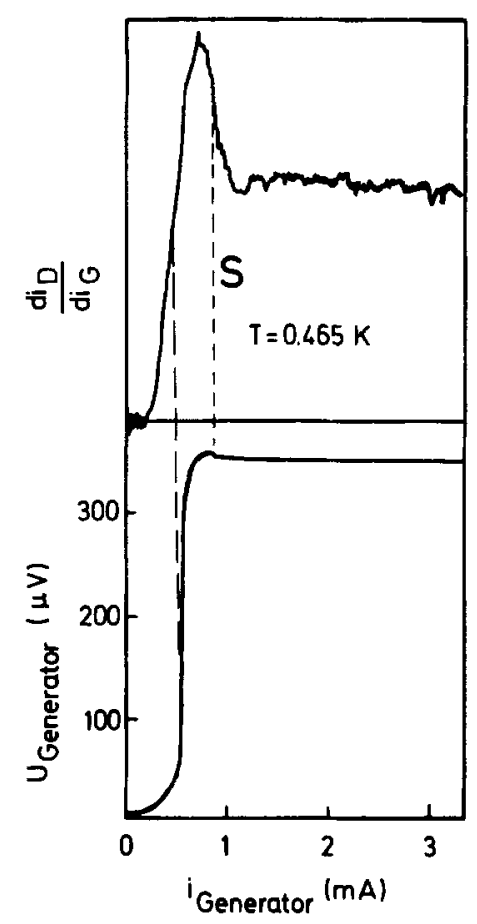

Fig. 12. Correlation between gap switching and reduction of the signal derivative (Diodes $1 \mathrm{a} \rightarrow \mathrm{b}$ )

In the phonon transmission detection experiments, marked consequences of the gap instabilities are seen (Fig. 11). The slope of the differentiated signal, observed in experiments with two equal pure low impedance diodes as generator and detector, shows all the structures, we identified above in the case $\Delta_{G 0}<\Delta_{D 0}$ (Fig. 11, comp. Fig. 4). The gap switching itself is connected with a reduction of the differentiated signal (Fig. 12). The corresponding curves

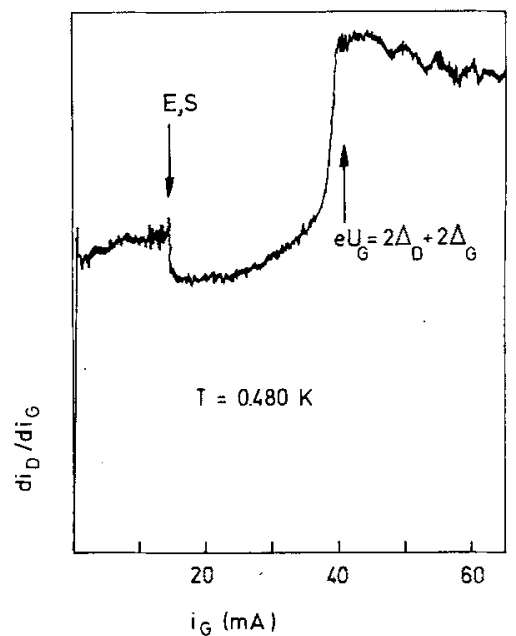

Fig. 13. Signal derivative $d i_{D} / d i_{G}$ for $\Delta_{G}>\Delta_{D}$ and slightly enhanced generator gap (Junctions $9 \mathrm{~b} \rightarrow \mathrm{a}$ )

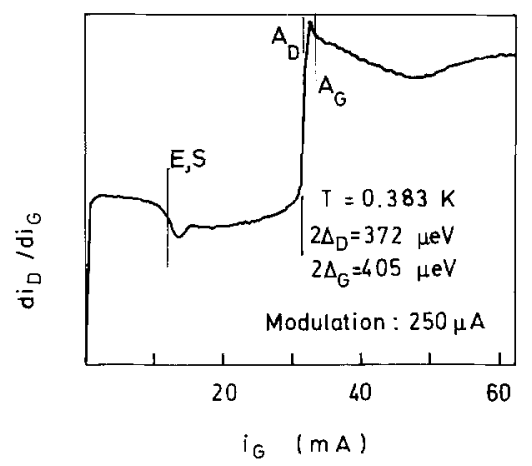

Fig. 14. Signal derivative $d i_{D} / d i_{G}$ for $\Delta_{G}>\Delta_{D}$ and slightly enhanced generator gap (Junctions $14 \mathrm{~b} \rightarrow \mathrm{a}$ )

with pure detector and a generator diode with slightly enhanced gap exhibit only this reduction of the differentiated signal (Fig. 13). Evidently, this degradation of the phonon yield is a consequence of the fact, that the recombination phonons partially do not fulfill the detection condition $\Omega \geqq 2 \Delta_{D}$. But it is remarkable, that in all cases observed, the shape of the phonon signal remained continuous at the gap switching point and only the differentiated signal showed either a sharp steplike reduction (Fig. 13) or a steplike reduction connected with a broad minimum (Fig. 14).

With regard to the phonon yield in experiments with low impedance tunneling generators, it was proposed in several works $[8,10]$ to explain the apparent nonlinearities by anomalies of the detector time constants. These models suggest, that the detector time constant $\tau_{\text {eff }}$ and thus the recorded signal can be enhanced by the injection conditions ("dynamical cooling" [10]). In order to exclude the possibility of such detector anomalies by clear experimental evidence, also the differential time constants 


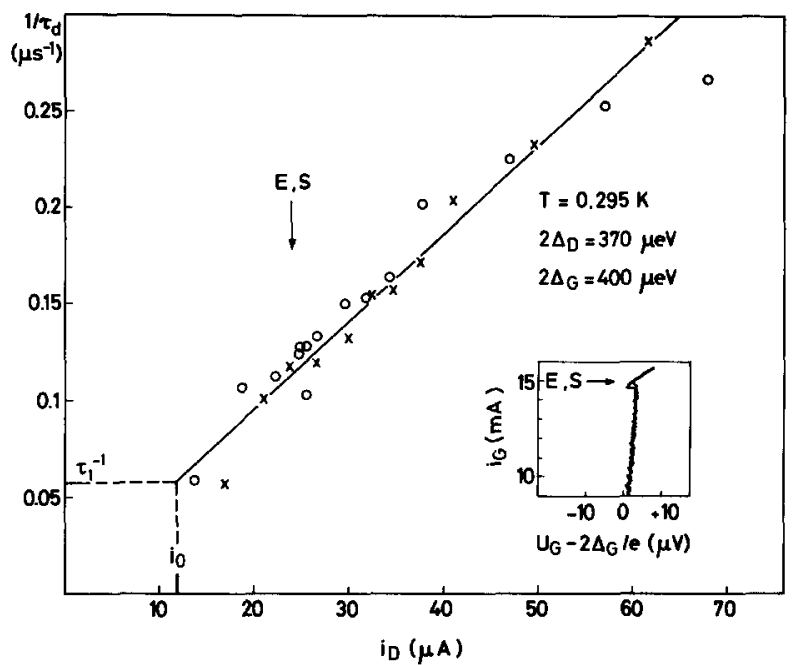

Fig. 15. Reduction of the detector time constant $\tau_{D}$ by injection (Diodes $9 \mathrm{~b} \rightarrow \mathrm{a}$ ). The crosses and circles refer to two different series of measurements

$\tau_{d}=d \rho / d J(J$ : quasiparticle injection rate) have been measured, which are directly relevant for the magnitude of the differentiated signal (comp. Sect. VI). The results of these measurements did not show in any case an anomal behavior like a bump or a sudden reduction also under extreme overinjection conditions (Fig. 15).

\section{Absolute Value of the Signal Amplitude}

If a tunneling detector is irradiated by the recombination phonons of a tunneling generator $\left(\Delta_{D}<\Delta_{G}\right)$ and the frequency of the recombination phonons does not exceed $4 \Delta_{D}$, then the theory predicts a signal amplitude of [28]:

$\delta i_{d}=2 \delta i_{G} T_{\perp D} i_{T D} \tau_{\mathrm{eff}} \bar{f} \bar{T}_{G S} \alpha /$

$\left(e \pi \rho_{T D} d r_{G D}^{2}\left(\bar{T}_{G S}+\bar{T}_{G H e}\right)\right)$

Thereby is: $i_{T D}=$ thermal detector tunneling current; $\rho_{T D}=$ thermal excitation density in the detector $\left(i_{T D} / \rho_{T D}\right.$ is a temperature independent constant of each detector) $\tau_{\text {eff }}=$ experimentally determined detector time constant; $\bar{f}=$ averaged phonon focusing factor; $d=$ total thickness of the detector films. The yield factor $\alpha$, which is defined by this formula, equals $\alpha=1$, if the following premises are fulfilled: (i) all recombination phonons are irradiated either into the substrate or into the He-bath, (ii) the angular distribution of the $k$-vectors in the substrate is given by Lambert's cosine law, and (iii) the detector behaves as quantum detector for phonons of energy $\Omega>2 \Delta_{D}$. All deviations from the theoretical

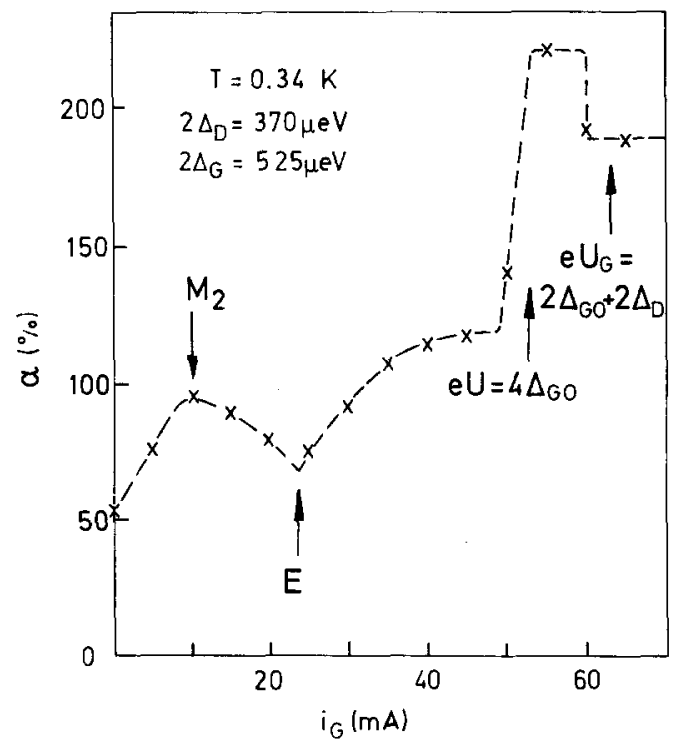

Fig. 16. Phonon yield factor for the combination of Diodes $8 b \rightarrow a$

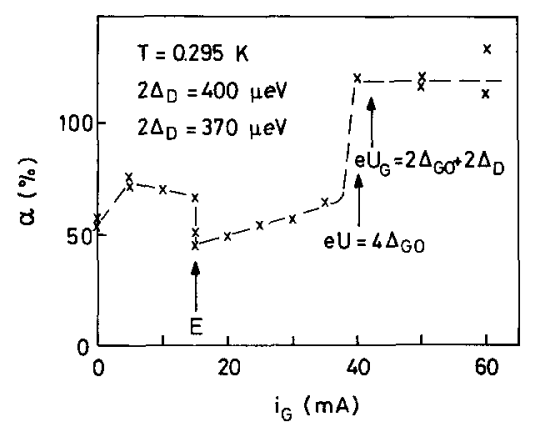

Fig. 17. Phonon yield factor for the combination of Diodes $9 b \rightarrow a$

predictions, such as phonon losses [28], are summarized in the additional yield factor $\alpha$.

Working with tunneling detectors and low impedance generators at low temperatures, the excess excitation density $\rho-\rho_{T}$ produced in the detector by phonon irradiation becomes often comparable to the thermal excitation density $\rho_{T}$. In this overinjection case, the relation (11) remains valid for small variations $\delta i_{G}$ and $\delta i_{D}$ provided that the time constant $\tau_{\text {eff }}=\left(\rho-\rho_{T}\right) / J$ ( $J=$ quasiparticle injection rate [15]) is substituted by $\tau_{d}(J)=d \rho / d J$. This differential time constant may directly be measured as a function of generator current $i_{G}$, by superimposing a small current pulse $\delta i_{G}$ to the $D C$-generator current; the pulse signal received decays with the time constant $\tau_{d}$.

If $\tau_{d}\left(i_{G}\right)$ and $\delta i_{D} / \delta i_{G}$ are experimentally determined, it is possible to calculate the yield function $\alpha\left(i_{G}\right)$ (Figs. 16-18). (Formally, also the absolute value of the relaxation phonon contribution is expressed in terms of an enhanced yield factor.) This calculation 


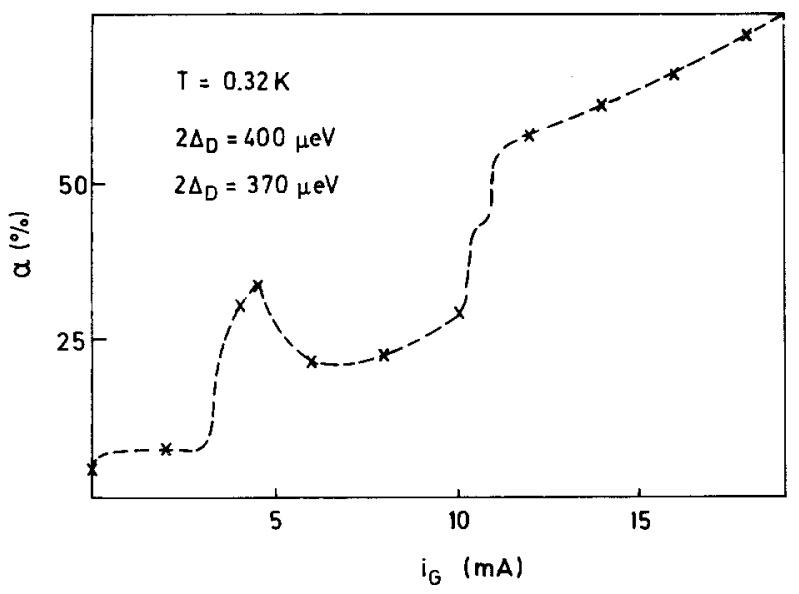

Fig. 18. Phonon yield factor for the combination of Diodes $9 a \rightarrow b$

has been carried out is using a transmission parameter $\bar{T}_{G H e}=0.2$. (Comp. Sect. V.)

In order to exclude experimentally a possible unknown influence of the overinjection in the detector, $\delta i_{D} / \delta i_{G}\left(i_{G}\right)$ was also recorded in using short pulses $(0.5 \mu$ s pulsewidth) as generator current. Provided that the inverse pulse repetition rate $(6 \mu \mathrm{s})$ is short compared to the detector time constant $\tau_{\text {eff }}(20 \mu \mathrm{s})$, the detector integrates the phonon pulses and the $D C$-signal may be analyzed and overinjection in the detector is avoided (Fig. 19).

In contrast to the results of similar measurements with Sn-diodes [2], the experimentally determined phonon yield is not constant in the range $2 \Delta_{G} \leqq e U_{G} \leqq 2 \Delta_{G}+2 \Delta_{D}\left(\Delta_{G}>A_{D}\right)$; (Figs. 16, 17), and evidently these anomalies are not a consequence of detector overinjection (Fig. 19). This discrepancy must be explained by the fact that the recombination phonon spectrum, emitted by superconducting films with a high phonon trapping factor $\tau_{\gamma} / \tau_{p}$ $(\mathrm{Sn}, \mathrm{Pb})$ is rather independent from the injection conditions and can be approximated by a quasithermal distribution $[16,20,21]$. On the other hand, the experimental results presented in Sect. II show the marked influence of the injection conditions on the spectrum of recombination phonons in the case of Al-diodes, and we further conclude from the observation of the gap reductions and instabilities (Sect. V) that also these effects are causing changes in the mean energy of the recombination phonons. Such variations of the energy of the emitted phonons are changing the phonon yield in three ways: (i) the recombination phonon energy may be smaller than the detector threshold $\Omega=2 \Delta_{D}$; this applies obviously in experiments with generators showing strong gap reductions or instabilities (Figs. 11-13). (ii) In Sect. $\mathrm{V}$ we discussed that due to the finite gap sharpness and the gap inhomogeneities, the pho-

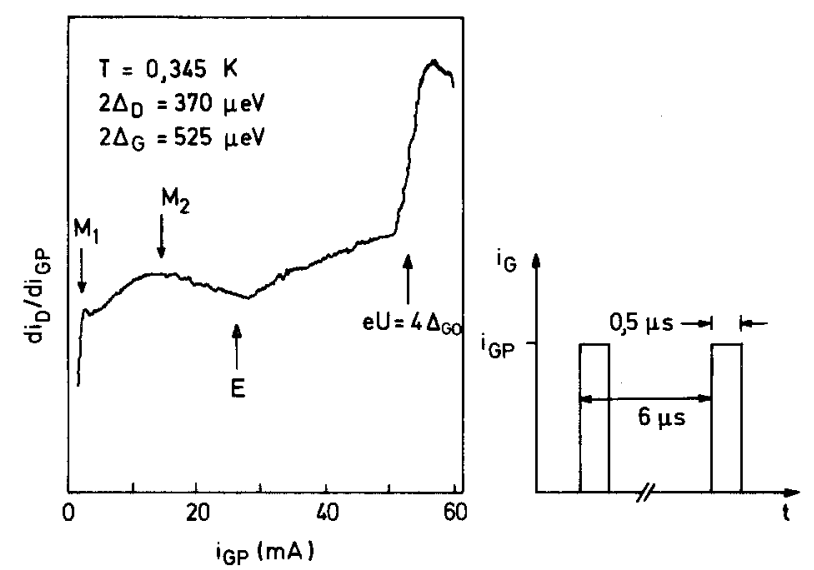

Fig. 19. Signal derivative of the Diodes $8 b \rightarrow a$. In order to avoid overinjection in the detector, the generator current was pulsed

non trapping factor may be changed by the injection conditions. Following the argumentation of Sect. IV, a change in the polarization of the emitted phonons and also in the measured phonon yield must be expected as a consequence of such variations of the trapping factor. Particularly under the focusing conditions of $c-\mathrm{Al}_{2} \mathrm{O}_{3}$, a cold quasiparticle distribution with a reduced trapping factor can result in an enhanced phonon yield. (iii) The theory predicts an approximately constant quantum response of a tunneling detector in the interval of phonon energy $2 A_{D} \leqq \Omega \leqq 4 A_{D}$ [6]. Nevertheless, the effective phonon yield can be influenced by the inelastic phonon decay processes, which seem to be a common feature of the metal insulator interfaces $[42,28]$. It must be assumed, that the primary phonons (energy $\Omega_{0}$ ) are partly converted by such inelastic processes in two or more low energetic phonons; the secondary phonon spectrum can cover the energy interval $0<\Omega<\Omega_{0}$. Reverting to the question of the recombination phonon yield, it follows that the detectable fraction of the secondary phonons should be strongly dependent on the energy of the primary recombination phonons. This extra detection of down-converted phonon was experimentally verified by Trumpp et al. [28]. (The observation of deep absorption lines in phonon spectroscopic experiments $[3,2]$ seems to be incompatible with the assumption of a high phonon decay rate. But in these experiments, not only the reduction of the phonon intensity at the resonant frequency is registrated, but also a reduction of the signal due to the resultant decay phonons.)

In fact, it is possible to explain qualitatively the observed anomalies within the frame work of the arguments given above. The increasing slope of the yield function $\alpha\left(i_{G}\right)$ in the interval $2 \Delta_{G}<e U_{G}<$ $2 \Delta_{G}+2 \Delta_{D}\left(\Delta_{D}<\Delta_{G}\right.$, Figs. 16, 17) must be considered 
Table 3. Phonon yield $\alpha$ determined with Al-junctions evaporated on $c-\mathrm{Al}_{2} \mathrm{O}_{3}$. The junctions $2 \mathrm{a}$ and $2 \mathrm{~b}$ exhibited an instability of the energy gap similar to junction 1 a (comp. Fig. 11, 12)

\begin{tabular}{lccccc}
\hline $\begin{array}{l}\text { Numbers of } \\
\text { the Junctions }\end{array}$ & $\begin{array}{c}2 \Delta_{\text {Go }_{0}} \\
\mu \mathrm{eV}\end{array}$ & $\begin{array}{c}2 \Delta_{D} \\
\mu \mathrm{eV}\end{array}$ & $\begin{array}{l}T \\
\mathrm{mK}\end{array}$ & $\begin{array}{c}\alpha \\
\%\end{array}$ & $\begin{array}{l}\alpha / \pi \\
\%\end{array}$ \\
\cline { 1 - 5 } $8 \mathrm{~b} \rightarrow \mathrm{a}$ & 525 & 370 & 340 & 52 & - \\
$9 \mathrm{~b} \rightarrow \mathrm{a}$ & 400 & 370 & 295 & 52 & - \\
$2 \mathrm{a} \rightarrow \mathrm{b}$ & 370 & 370 & 419 & 13 & 17 \\
$2 \mathrm{~b} \rightarrow \mathrm{a}$ & 370 & 370 & 424 & 11.5 & 15.4 \\
$9 \mathrm{a} \rightarrow \mathrm{b}$ & 370 & 400 & 308 & 5.1 & 14 \\
& & & 318 & 5.9 & 15 \\
& & & 363 & 8.0 & 18 \\
& & & 479 & 15 & 26 \\
& & & 511 & 20 & 33 \\
$8 \mathrm{a} \rightarrow \mathrm{b}$ & 370 & 525 & 294 & 0.30 & 68 \\
& & & 337 & 0.52 & 54 \\
& & & 359 & 0.61 & 45 \\
& & & 460 & 0.40 & 99 \\
\hline
\end{tabular}

as a consequence of the increasing mean frequency of the recombination phonons connected with enhanced extra detection of secondary phonons. The reasons for this broadening of the recombination phonon spectrum are the injection of a broad quasiparticle distribution at higher generator voltages and an increase of the direct recombination processes at high excitation density. (In principle, also a possible contribution of the low energetic relaxation phonons to the detector signal must be discussed; but this possibility may be ruled out with regard to the long mean path of such phonons $\left(\Omega<2 \triangle_{D}\right)$ in the detector films at low temperatures [14]). The interpreation of the structures in the range $e U_{G}=2 \Delta_{G}$ is not so unambiguous, but it is plausible to explain the two maxima $\left(M_{1}, M_{2}\right.$, Figs. 16,19$)$ as states with a reduced effective phonon trapping factor due to the finite sharpness of the gap and to inhomogeneities, respectively (comp. Sect. IV, V). Further, all gap instabilities of the generator diodes are connected with a strong reduction of the recombination phonon yield (Fig. 17, Structure $E, S$ ).

Under the condition $\Delta_{G}>\Delta_{D}$ a yield factor of $\alpha=52 \%$ was obtained in the low injection regime; this value describes not only the yield of ballistic phonons but also the contribution of sidewall scattered ones. From time resolved measurements performed with the same crystal $\left(c-\mathrm{Al}_{2} \mathrm{O}_{3}, 3 \mathrm{~mm}\right.$ thickness, $10 \mathrm{~mm}$ diameter) we know that the ratio of the sidewall reflected signal to the ballistic signal is $\alpha_{S W} / \alpha_{B} \cong 0.12$ and conclude that the yield of ballistic phonons is $\alpha_{B} \cong 46 \%$. The error, resulting from the limited accuracy of the experimental values, is $\pm 30 \%$. Comparing this with the values reported by Trumpp et al. on corresponding experiments with tin diodes on $\mathrm{Si}$ [28] we state that the losses of
$2 \Delta_{\mathrm{Sn}}$-phonons are significantly higher: the values of the yield factor $\alpha_{B, 2 \Delta_{\mathrm{S}}}$ range from $8 \%$ to $13 \%$, depending significantly on the preparation conditions of the Si-surfaces [28]. Further, also the reanalysis of unpublished measurements [43] of the phonon signal amplitude, determined with tin diodes on $\mathrm{Al}_{2} \mathrm{O}_{3}$, yields the value of $\alpha_{B, 2 \Delta_{\mathrm{Sn}}} \cong 10 \%$. Since the main loss source must be seen in the decay processes of high frequency phonons [28], we conclude that the comparatively higher phonon yield in the case of $2 \Delta$-Al-phonons is due to the lower frequency of the recombination phonons and to the frequency dependence of the so far unknown decay processes. $\left(2 \Delta_{\mathrm{A} 1}=400 \mu \mathrm{V}\right.$ corresponds to $90 \mathrm{GHz}$. Following the hypothesis that the decay processes are located in the metal-insulator boundaries generator - substrate and substrate - detector, the losses in one boundary are appr. $33 \%$ and $64 \%$ for phonons having a frequency of $90 \mathrm{GHz}$ or $280 \mathrm{GHz}$, respectively.

In Table 3 also the temperature dependence of the phonon yield measured in experiments with $\Delta_{G}<\Delta_{D}$, $e U_{G}=2 \Delta_{G}$ is reported. Assuming the quasithermal model of Long [16], the recombination spectrum is $N(\Omega) \sim \Omega^{2} \cdot \exp (-\Omega / k T)\left(\Omega>2 \Delta_{G}\right)$ and for $\Delta_{G}<\Delta_{D}$ the phonon yield should be $\alpha=\alpha_{0} \Pi$ with

$$
\Pi\left(\Delta_{G}, \Delta_{D}\right)=\int_{2 \Delta_{D}}^{\infty} N(\Omega) d \Omega / \int_{2 \Delta_{G}}^{\infty} N(\Omega) d \Omega
$$

In contrast to this model the measured values (see Table 3) are much smaller in the case of low temperature and small gap differences $\Delta_{p}-\Delta_{G}$. This indicates that working in the high injection range and biasing the detector at $e U_{G}=2 \Delta_{G}$ the produced quasiparticle and phonon distributions can be narrower than predicted by the quasithermal model.

\section{Conclusions}

The above experiments demonstrate that the recombination phonon spectrum emitted by superconducting Al-junctions cannot be described adequately by a quasithermal function. The spectrum shows sharp structures due to the nonthermalized phonons generated in the first decay steps of the excess quasiparticles injected by tunneling. The observation of these structures confirms the validity of the developed models for the phonon generation in superconducting films $[12,13,7,8]$. At generator voltages $2 \Delta_{G}<e U_{G}<4 \Delta_{G}$ a sharp peak at the phonon energy $\Omega=e U_{G}$ is the dominant feature of the recombination spectrum of tunneling diodes with low phonon trapping. The inadequacy of a qua- 
sithermal model is further confirmed by the temperature dependence of the phonon yield in experiments with $\Delta_{G}<A_{D}$.

Considering low impedance generators, the recombination phonon yield is nonlinear in accord with earlier measurements $[8,10]$. It is shown, that these nonlinearities are caused by gap reductions and inhomogeneities of the generator films under injection. The absolute value of the recombination phonon yield indicates that phonon loss processes are by about a factor of 4 weaker in the case of Al-recombination phonons $(100 \mathrm{GHz})$ than in the case of $280 \mathrm{GHz}$ phonons [28].

Finally, it was observed that the relaxation phonon contribution at $e U_{G}=4 A_{G}$ (reabsorption threshold) is dependent on the substrate and the film preparation. These facts may be qualitatively explained by the different trapping conditions for longitudinal and transverse phonons. The experimental results are quantitatively compared with the predictions of a two-modes model and the possibility of mode converting processes in the superconducting films is discussed.

We gratefully acknowledge discussions with Dr. K. Lassmann, Dr. H.-J. Trumpp and Dr. W. Forkel. Financial support granted by the "Deutsche Forschungsgemeinschaft" is gratefully acknowledged.

\section{References}

1. Eisenmenger, W., Dayem, A.H.: Phys. Rev. Lett. 18, 125 (1967)

2. Kinder, H.: Phys. Rev. Lett. 28, 1564 (1972)

3. Kinder, H.: J. Phys. 33, C4-21 (1972)

4. Welte, M., Lassmann, K., Eisenmenger, W.: J. Phys. 33, C4-25 (1972)

5. Forkel, W., Welte, M., Eisenmenger, W.: Phys. Rev. Lett. 31, 215 (1973)

6. Eisenmenger, W.: In: Physical acoustics. Mason, W.P., Thurston, R. (eds.), Vol. XII, p. 79. 1976

7. Kinder, H., Lassmann, K., Eisenmenger, W.: Phys. Lett. 31 A, $475(1970)$

8. Dayem, A.H., Wiegand, J.J.: Phys. Rev. B5, 4390 (1972)

9. Dayem, A.H.: J. Phys. 33, C4-15 (1972)

10. Long, A.R., Adkins, C.J.: Phil. Mag. 27, 865 (1973)

11. Giaever, I., Megerle, K.: Phys. Rev. 122, 1101 (1961)

12. Tewordt, L.: Phys. Rev. 127, 371 (1962)
13. Tewordt, L.: Phys. Rev. 128, 12 (1962)

14. Bobetic, V.M.: Phys. Rev. 136, 1535 (1964)

15. Rothwarf, A., Taylor, B.N.: Phys. Rev. Lett. 19, 27 (1967)

16. Long, A.R.: J. Phys. 33, C4-73 (1972)

17. Trumpp, H.J., Lassmann, K., Eisenmenger, W.: Phys. Lett. 41 A, 431 (1972)

18. Eisenmenger, W., Lassmann, K., Trumpp, H.J., Krauss, R.: Appl. Phys. 11, 307 (1976)

19. Eisenmenger, W., Lassmann, K., Trumpp, H.J., Krauss, R.: Appl. Phys. 12, 163 (1977)

20. Owen, C.S., Scalapino, D.J.: Phys. Rev. Lett. 28, 1559 (1972)

21. Parker, W.H.: Phys. Rev. B12, 3667 (1975)

22. Aronov, A.G., Spivak, B.Z.: J. Low Temp. Phys. 29, 149 (1977)

23. Abeles, B., Cohen, R.W., Cullen, G.W.: Phys. Rev. Lett. 17, 632 (1966)

24. Cohen, R.W., Abeles, B.: Phys. Rev. 168, 444 (1968)

25. Welte, M.: Thesis, Stuttgart 1976

26. Chang, J.J., Scalapino, D.J.: J. Low Temp. Phys. 31, 1 (1978)

27. Pokrovskii, V.L.: Sov. Phys. JETP 13, 628 (1961)

28. Trumpp, H.J., Eisenmenger, W.: Z. Phys. B - Condensed Matter 28, 159 (1977)

29. Taylor, B., Maris, J.H., Elbaum, C.: Phys. Rev. Lett. 23, 416 (1969)

30. Weiss, O.: Private Communication (1976)

31. Elbaum, C.: International Conference on Phonon Scattering in Solids Albany, H.J. (ed.), 3-6 July 1972, Paris, p. 1

32. Kinder, H., Dietsche, W.: In: Phonon Scattering in Solids. Challis, L.J., Rampton, V.W., Wyatt, A.F.G. (eds.), p. 199, (1976)

33. Long, A.R.: J. Phys. F 3, 2023 (1973)

34. Trumpp, H.J.: Thesis, Stuttgart 1976 (unpublished)

35. Mühlschlegel, B.: Z. Phys. 155, 313 (1959)

36. Welte, M.: Z. Phys. B - Condensed Matter 29, 107 (1978)

37. Chang, J.J., Scalapino, D.J.: Phys. Rev. B 10, 4047 (1974)

38. Dynes, R.C., Narayanamurti, V., Garno, J.P.: Phys. Rev. Lett. 39, 229 (1977)

39. Gray, K.E., Willemsen, H.W.: J. Low Temp. Phys. 31, 911 (1978)

40. Döttinger, S.: (Private Communication)

41. Kinder, H., Dietsche, W.: Phys. Rev, Lett. 33, 578 (1974)

42. Day, W.: J. Phys. 33, C4-65 (1972)

43. Kinder, H., Lassmann, K., Eisenmenger, W.: Akkustische Quantenprozesse in Supraleitern. 1969 (unpublished)

44. Kinder, H.: Z. Phys. 262, 295 (1973)

M. Welte

W. Eisenmenger

Physikalisches Institut

- Teilinstitut 1 -

Universität Stuttgart

Pfaffenwaldring 57

D-7000 Stuttgart 80

Federal Republic of Germany 